\title{
LA MEMORIA COLECTIVA Y LAS TENSIONES ESENCIALES DEL (BUEN) HISTORIADOR (DE LA CIENCIA)
}

\author{
Fernando BRONCANO
}

\begin{abstract}
"Es una revelación cotejar el don Quijote de Menard con el de Cervantes. Este, por ejemplo, escribió (Don Quijote, primera parte, noveno capítulo):

...la verdad, cuya madre es la historia, depósito de las acciones, testigo de lo pasado, ejemplo y aviso de lo presente, advertencia de lo porvenir.

Redactada en el siglo diecisiete, redactada por el "ingenio lego" Cervantes, esa enumeración es un mero elogio retórico de la historia. Menard, en cambio, escribe:

...la verdad, cuya madre es la historia, depósito de las acciones, testigo de lo pasado, ejemplo y aviso de lo presente, advertencia de lo porvenir.

La historia, madre de la verdad; la idea es asombrosa. Menard, contemporáneo de William James, no define la historia como una indagación de la realidad sino como su origen" ("Pierre Menard, autor del Quijote", J.L. Borges).
\end{abstract}

Kuhn ha contribuido más que ningún filósofo de la segunda mitad del siglo a configurar nuestra visión de la ciencia contemporánea. En este trabajo nos vamos a referir a un aspecto muy limitado de su obra, aunque no al menos importante, a la fundamentación de la disciplina a la que él siempre consideró que pertenecía, a la Historia de la Ciencia. No se trata de una mera reivindicación de autonomía profesional que pueda explicarse por causas sociológicas sino que Kuhn apunta a un problema intelectual de primer orden, el de cómo es posible recuperar la memoria colectiva de nuestro pasado cognitivo a partir de un registro histórico lingüístico y parcial y quizás esencialmente oculto. La cuestión que plantea Kuhn, desde nuéstro punto de vista, es la de cómo es posible la memoria de un proceso que tiene de manera intrínseca a) una estructura histórica y b) en tanto que proceso

Éndoxa: Series Filosóficas, $n^{\circ} 9,1997$, UNED, Madrid:

Fernando Broncano: La memoria colectiva y las tensiones esenciales del (buen) historiador (de la ciencia).

pp. 51-75. 
cognitivo, una existencia opaca, en el sentido quineano, a saber, el descubrimiento científico. En resumen, cómo es posible la Historia de la Ciencia como un fin en sí mismo.

\section{La historia en la ciencia}

La ciencia es un sistema social de producción de conocimientos uno de cuyos motores es la curiosidad por lo desconocido y el otro la lucha por el reconocimiento inter pares. Científicos a quienes no imaginariamos cruzando un semáforo en rojo son capaces del peor comportamiento moral para conseguir el reconocimiento que a sus ojos merecen por parte de la comunidad de su disciplina; individuos quizás no muy preocupados por los intereses mundanos competirán como organismos darwinianos para transmitir sus ideas propias en la espera de que sean reconocidas, aceptadas, usadas, citadas, admiradas. No importa la escala de observación que elijamos; la actividad de las comunidades científicas está muy lejos de la imagen del pacífico sistema de la burocracia weberiana, recuerda más el espectáculo de una plurisilva que el de un jardín racionalista.

Un sistema basado en el reconocimiento no puede ser insensible a la historia, es, por el contrario, un sistema esencialmente mitopoyético, generador de historias heroicas de pioneros $y$ descubridores. La historia épica de la ciencia es uno de los recursos más efectivos del sistema para el reclutamiento de nuevos miembros de la comunidad. La historia es, al tiempo, un sistema de primer orden para el mantenimiento de la propia identidad de la comunidad como empresa colectiva. El sistema es, pues, un consumidor de historia o, mejor, de "historias". El propio sistema es, además, histórico en un sentido m!s profundo, su estructura constitutiva contemporánea es fruto de trayectorias pasadas contingentes de descubrimientos, institucionalizaciones y generación de habilidades que hacen de la ciencia en cada momento un sistema único e irrepetible. 
El caso es que la historia de la ciencia funciona en cierta medida como parte del propio proceso de desarrollo de la ciencia. En tanto que memoria registrada por parte de los investigadores en activo, tiende a sufrir los mismos avatares que la propia dinámica de la investigación, en períodos de ciencia normal se convierte en registro histórico de descubrimientos y, en períodos de ciencia revolucionaria, se aplica la reescritura orwelliana de la historia: quienes tienen el poder reescriben el pasado como un proceso que conduce inevitablemente a su sitio en la historia. Tales procedimientos no tienen nada de malo ni de bueno, no afectan de manera importante al contenido ni a la evaluación de la teoría, sino que son hábitos que tienen sentido dentro de la red de actos retóricos que constituye la vida cotidiana de comunicación y competencia de las comunidades. Si las concesiones de crédito son lo que más importa al investigador, éste usará las concesiones del pasado como piedras millares que orientan su camino hasta el lugar donde se encuentra. No tiene mucha importancia si las asignaciones de crédito fueron merecidas o no, si hubo o no vías alternativas que quedaron ocultas, al investigador solamente le interesa el apoyo de la autoridad del pasado inmediato para justificar sus resultados presentes.

Pero no es algo que el historiador profesional acepte de buen grado: a el no le preocupa, o no debería hacerlo, la utilidad de su reconstrucción, le preocupa su veracidad. La tesis de Kuhn es que la representación histórica, al igual que su justificación abstracta por los filósofos de la ciencia ${ }^{1}$, es una representación esencialmente sesgada, que oculta sistemáticamente la práctica real de la ciencia, una práctica que, piensa Kuhn, debe ser reconstruida con métodos autónomos.

\footnotetext{
${ }^{1}$ Ahora y en adelante consideraremos (caritativamente) que la Filosofía de la Ciencia es parte de, o se reduce a, la autoimagen media de la ciencia en cada momento histórico, que se expresa de manera abstracta en un vocabulario profesional específico. La cuestión es más complicada, es cierto, pero para nuestros propositos actuales es suficiente.
} 
Así que nos encontramos con la paradójica y posible situación de que el trabajo histórico, en la medida que es útil, es o puede ser falso $y$ en la medida en que es verdadero es o puede ser inútil. ¿Para qué, pues, la Historia de la Ciencia?

\section{Un mundo de historias}

Kuhn ve su propia obra como un alegato en favor de una nueva historiografía y para dejar clara su misión comienza así $L a$ Estructura de las revoluciones cientificas

"Si se considera a la historia como algo más que un depósito de anécdotas o cronologia, se puede producir una transformación decisiva de la imagen que tenemos actualmente de la ciencia." (Kuhn, 1970, p. 20)

Hay dos historiografías. La vieja historiografía está generada por una imagen falsa de la ciencia y quienes la practican contribuyen a extender y asentar la imagen falsa. Son los científicos, por causas que acabamos de expresar y los filósofos, por razones menos confesables. En ambos casos Kuhn diagnostica una característica común: la Historia de la Ciencia es un medio para otros fines, no un fin en sí misma. Los fines se establecen desde fuera, en el caso de los científicos, dice Kuhn,

"además de su atractivo intrínseco, un medio de aclarar los conceptos de su especialidad, de establecer su tradición y de ganar adeptos." (Kuhn, 1968, p. 129)

En el caso de los filósofos la Historia no es más que un medio para mostrar ejemplos de tesis filosóficas a priori sobre el método científico, el conocimiento, la naturaleza de la ciencia, etc. La vieja historiografía, además, sea en la versión espontánea de los científicos y filósofos o en la profesional de los historiadores influidos por aquéllos, nace con un pecado original: se basa en la asimilación del pasado al presente. Los viejos historiadores proyectan 
el conocimiento contemporáneo sobre el pasado de dos formas principales: en primer lugar seleccionando unos materiales históricos sobre otros, en segundo lugar asignando más o menos importancia histórica a unos materiales que a otros. La selección y la evaluación, por consiguiente, son las formas en las que se expresa el sesgo que la imagen legendaria de la ciencia produce en la Historiografía. El resultado final es una conversión de lo que es un proceso contingente y sometido a bruscas y profundas transformaciones en un proceso acumulativo, que parece conducir de manera necesaria a lo que en apariencia sería su fin necesario, el del status quo cuando se escribe la historia.

¿Cuál sería pues el papel de la nueva historiografía?, al menos, ¿cuál sería su utilidad, si es que llega alguna vez a construir una imagen más adecuada de la ciencia? Kuhn habla esporádicamente de un efecto pedagógico, de influencia sobre los filósofos, pero no manifiesta tener una respuesta rápida y segura a la pregunta, lo que, paradójicamente, me parece que es el mejor signo, aunque sea un signo ad hominem, de que Kuhn se ha tomado en serio su propia disciplina. Si le preguntamos a un científico cualquiera cuál es la función de las matemáticas nos entretendrá con una larga lista, pero si le dirigimos la misma pregunta a un matemático solamente suscitaremos en él una cara de perplejidad. ¿Cuál es el papel de la memoria? ¿Cuál es el papel de la memoria colectiva cognitiva? Me parece que es un signo de buena salud no encontrar ninguna respuesta fácil a la pregunta. Cualquier otra nos conduce seguramente de nuevo a la senda de la instrumentalización.

El descubrimiento es un proceso esencialmente histórico, y a tal evento, esencialmente singular, contingente, contextual, cambiante, le corresponde un producto intelectual específico, que no es una teoría, como es el caso de la ciencia, ni un argumento, como ocurre en la filosofía, sino que son narraciones, como lo que produce el historiador, pero son narraciones de un tipo muy especial: representan un proceso que es en parte interno (y por ello depende de la dinámica de los modelos mentales de un individuo) y en 
parte depende del marco teórico que el individuo ha heredado de la comunidad a la que pertenece. Cuándo la narración es buena y cuándo es mala depende de factores muy sutiles que son precisamente los que hacen de la Historiografía de la Ciencia una práctica con sus propios compromisos evaluativos.

"El producto final de la mayor parte de la investigación histórica es una narración de hechos particulares del pasado. Es, en parte, una descripción de lo que ocurrió - una mera descripción suelen decir los filósofos y científicos-Su éxito depende, sin embargo, no sólo de la exactitud sino también de la estructura. La narración histórica debe hacer plausibles y comprensibles los acontecimientos que describe. En cierto sentido [...] la historia es una empresa explicatoria; $y$, a pesar de ello, logra sus funciones explicatorias sin recurrir casi a generalizaciones explícitas". (Kuhn, 1976, p. 29)

La estructura de la narración es especial, a medio camino entre la historia de las ideas y la historia general ${ }^{2}$. Y por si hubiera alguna confusión respecto a qué tipo especial de narraciones produce la Historia de la Ciencia, nos recuerda Kuhn un poco más tarde,

"la actividad del historiador no puede compararse a la del niño que arma rompecabezas cuyas piezas están contadas; el historiador recibe muchas piezas extra. Tiene o puede obtener los datos, no todos (¿qué sería eso?), pero sí una extensa colección. Su trabajo consiste en seleccionar de aquí un conjunto que pueda yuxtaponerse de manera que sus elementos formen lo que, en el caso del niño, sería una imagen de objetos reconocibles, organizados coherentemente, y lo que, para el historiador y sus lectores, es una narración plausible que envuelve motivos y conductas reconocibles". (Kuhn, 1976, p. 41)

De manera que el historiador se convierte en alguien que nos proporciona explicaciones de hechos que están en el registro histórico mediante "una narración plausible que envuelve motivos y conductas reconocibles". Es otra forma de decir que el historia-

${ }^{2}$ Kuhn, 1971 es el artículo complementario de Kuhn, 1976, en lo que se refiere a la distancia especial que el historiador de la ciencia tiene respecto al historiador, y por extensión, respecto al sociólogo. 
dor nos da explicaciones de hechos que tienen la naturaleza de lo que los filósofos consideran explicaciones intencionales, aunque sea de un tipo especial de hechos que llamamos "descubrimientos". Pero una vez que hemos descubierto el Mediterráneo, no siempre reconocido por los lectores y críticos de Kuhn, es cuando comienzan las cuestiones más interesantes.

\section{La narración y el alcance del relativismo}

La metodología kuhniana de la Historia de la Ciencia ha generado, en una autoaplicación de sus propias tesis, una magna polémica de carácter filosófico sobre el alcance del relativismo como consecuencia inevitable del propio trabajo histórico. La cuestión del relativismo aparece en varios planos, el más popular es el del la epistemología y metodología. Puesto que científicos que trabajan en diferentes paradigmas viven en mundos diferentes, suele afirmar Kuhn, el cambio revolucionario resulta "inconmensurable" desde el punto de vista de la evaluación de la racionalidad de las decisiones adoptadas por los científicos implicados. Mucho más fundamental para nuestro tema de la escritura de narraciones es otro aspecto de la cuestión estrechamente unido a la inconmensurabilidad. Se trata de la "intraducibilidad" de los lenguajes en que están expresados los dos paradigmas. Kuhn y Feyerabend argumentaron, siguiendo a Sapir y Lee Whorf, en favor de la carga teórica de los significados. Al cambiar las teorías cambian los significados de los términos que las expresan, de manera que es imposible reconstruir una teoría en el lenguaje de otra, sea por imposibilidad absoluta, sea, como defiende Kuhn, a causa de que se pierde significado sustancial de los términos más importantes que categorizan el mundo. De manera que la expresión "vivir en mundos diferentes" tiene ahora un apoyo lingüístico. Sobre esta base plantea Kuhn las cuestiones nucleares acerca de las peculiaridades metodológicas de la Historia de la Ciencia, puesto que al llevar la hermenéutica a la arena del lenguaje, varias de las 
discusiones ya clásicas sobre fundamentos de las ciencias humanas, de las que la obra de Kuhn es heredera, adquieren una luz nueva, esencialmente nueva.

El trabajo más conocido que abrió esta rama de la discusión es el de Davidson, 1974, "Sobre la idea misma de un esquema conceptual". En este trabajo Davidson acusaba al relativismo epistémico basado en el relativismo lingüístico de ser esencialmente fiel a la tradición empirista que pretenden denostar. Davidson argumenta que el relativismo está comprometido con lo que denominó el "tercer dogma" del empirismo, tras el dogma de la reducibilidad del significado al contenido empírico y del segundo dogma de la distinción entre enunciados analíticos y sintéticos, que Quine ha incorporado para siempre a la tradición filosófica. En palabras de Davidson, este tercer dogma aparece cuando

"en lugar del dualismo de lo analítico-sintético tenemos el dualismo de esquema conceptual y contenido empírico. El nuevo dualismo crea los cimientos de un empirismo al que se han amputado los dogmas insostenibles de la distinción analítico-sintética y del reduccionismo, aliviándolo, asi, de la idea impracticable de que podemos asignar un contenido empírico individualmente, oración por oración." 3

Este nuevo dogma lo encuentra Davidson en la tesis del relativismo lingüístico de Sapir y Lee Whorf, para quienes el lenguaje, como organizador de la experiencia,

"presenta un nuevo principio de relatividad, que sostiene que los observadores no están todos guiados por la misma evidencia física hacia la misma ilustración del universo, a menos que sus encuadres lingüísticos sean similares, o que puedan ser calibrados de alguna manera."

El dualismo se presenta entre dos polos

Davidson, 1974, p. 195.

${ }^{4}$ B.L. Whorf, "The Punctual and Segmentative Aspects of Verbs in Hopi" en Language, Though and Reality: Selected Writing of Benjamin Lee Whorf, Cambridge, Mass: MIT Press, cit. en Davidson, 1974, p. 195. 
"el lenguaje, como fuerza organizadora, que no se distingue claramente de la ciencia; y lo que es organizado, referido de varias maneras como "experiencia", "el flujo de experiencia sensorial", y "evidencia física."5

En medio de un cambio teórico sustancial, como el que ocurre en las revoluciones científicas, percibiríamos el proceso como imposibilidad de traducir el viejo lenguaje al nuevo, de vivir simultáneamente la vieja y la nueva experiencia.

Una popular metáfora relativista ha sido la del cambio de Gestalt, y a esta intelección han colaborado los ejemplos wittgensteinianos de la doble imagen "pato-conejo" empleada sistemáticamente por Hanson y no negada por Kuhn al principio de la polémica. Pero esta metáfora es precisamente el objeto de la justificada crítica de Davidson. El argumento de Davidson es que, cuando concebimos el lenguaje como "organizador" de la experiencia y al tiempo pensamos en lenguajes completamente intraducibles, las nociones de lenguaje y de mundo resultan ambas simple y completamente ininteligibles. $Y$ además son los dos polos de la relación los que resultan ininteligibles. Un lenguaje supuestamente imposible de traducir, aduce Davidson, no sería considerado como lenguaje, la conducta de habla no sería considerada como conducta de habla, la escritura sería no más que puro grafismo, y el otro polo, la experiencia, o cualquiera de sus sustitutos, sería absolutamente imposible concebirla independientemente de un lenguaje. Sería como hablar de lenguajes y experiencias en el mismo sentido que hablamos del lenguaje de las abejas y de la experiencia de los cuervos volando,

"No podemos asignar un significado claro a la noción de organizar un único objeto (el mundo, la naturaleza, etcétera) a menos que se entienda que el objeto contiene o consiste en otros objetos. Alguien que se pone a organizar un armario ordena las cosas que hay en su interior. $\mathrm{Si}$ a usted le dijeran que no organizara los zapatos y las camisas, sino el armario, usted se quedaría

${ }^{5}$ Davidson, 1974, p. 195. 
perplejo. ¿Cómo organizaría usted el Océano Pacífico? Enderezaría sus costas tal vez, o reubicaría sus islas, o destruiría sus peces."

Y sin embargo esta forma de relativismo es la más popular en las discusiones de café, en las que la metáfora de ver el mundo de forma distinta se emplea irrestricta e inconsecuentemente. Kuhn se ha quejado de que no es eso lo que el quería expresar, aunque reconoce una parte de culpa:

"casi nadie se ha enfrentado por completo con los problemas que nos indujeron, a Feyerabend y a mí, a hablar de inconmensurabilidad. No hay duda de que ese descuido se debe, en parte, al papel desempeñado por la intuición y la metáfora en nuestras formulaciones iniciales. Por ejemplo, yo utilizaba mucho el verbo "ver", y asemejaba una y otra vez los cambios de teoría a los cambios de Gestalt." (Kuhn, 1983, p. 97)

En escritos recientes Kuhn se ha ido distanciando del uso "metafórico del término "inconmensurabilidad". Motivado por esta crítica, Kuhn adopta una perspectiva más matizada de fracaso local y no global en la traducibilidad de los términos:

"Cuando se aplica al vocabulario conceptual que se da en una teoría científica y en su entorno, el término "inconmensurabilidad" funciona metafóricamente. La frase "sin medida común" se convierte en "sin lenguaje común" [...] La mayoría de los términos comunes a las dos teorías funcionan de la misma forma en ambas; sus significados, cualesquiera que puedan ser, se preservan; su traducción es simplemente homófona. Surgen problemas de traducción únicamente con un pequeño subgrupo de términos (que usualmente se interdefinen) y con los enunciados que los contienen. La afirmación de que dos teorías son inconmensurables es más modesta de lo que la mayor parte de sus críticos y críticas ha supuesto." (Kuhn, 1983, p. 99).

Esta afirmación de modestia parece alejarse definitivamente de la amenaza de la crítica davidsoniana de un lenguaje completamente ininteligible y una experiencia completamente ajena, y

${ }^{6}$ Davidson, 1974, p. 197. 
parece convertirla en una mera caricatura de Kuhn, fruto del malentendimiento. Pero, ¿evita la no traducibilidad local el argumento davidsoniano? No, desde el punto de vista de Davidson, y su respuesta nos lleva a otro estrato más profundo en el trabajo del historiador.

Traducibilidad, comunicación y "caridad" en la interpretación

El historiador narra mundos perdidos en el tiempo, del mismo modo que el científico en medio de un cambio revolucionario se enfrenta a mundos ajenos en el espacio social. En ambos casos tenemos un sentido abierto de la enajenación: es otra gente, otra comunidad, otra conducta, y un sentido opaco: es otro esquema conceptual, otro lenguaje, otro significado. De ahí que sea fácil entender que si son absolutamente otros será absolutamente imposible tanto la tarea del historiador como la discusión científica. El reemplazo de un paradigma por otro se convierte en algo muy sabido por cualquier historiador o antropólogo, no tiene mayor significación que la invasión de una comunidad por otra. Al fin y al cabo Gengis Khan no necesitaba entender a los chinos.

Pero no es esto lo que quiere expresar Kuhn con una revolución científica, el piensa más bien que un fracaso local de la traducción presenta al historiador de las ideas un enigma metodológico de primer orden:

"Si algunos términos no vacuos de una teoría antigua eluden la traducción al lenguaje de su sucesora, ¿cómo pueden los historiadores y demás analistas tener tanto éxito al reconstruir esa teoría más antigua, incluyendo el uso y función de aquéllos mismos términos?"7

Davidson se hace la misma pregunta y su respuesta es que a Kuhn no le salva de la acusación de incoherencia esta retirada a la traducción parcial. El argumento de Davidson es relevante para el

\footnotetext{
${ }^{7}$ Kuhn, 1983, p.101.
} 
problema del historiador autónomo. Según Davidson la tarea de traducir un lenguaje ajeno y la de interpretar o entender los significados de otras personas, dada su conducta verbal, son tareas indisolublemente unidas. Nos encontramos ante la tríada formada, en primer lugar, por el contexto abierto de la evidencia empírica compartida, en segundo lugar, por las creencias internas del hablante, o el contenido de su estado mental si se quiere, $y$, en tercer lugar, por el significado de su conducta verbal. En casos normales y cercanos de conversación raramente acudimos a las creencias del hablante para entender su conducta verbal, o sólo lo hacemos cuando esa conducta nos sorprende o nos resulta ambigua. Pero siempre, sea normal o no el contexto, se trata de resolver una ecuación con dos incógnitas, qué es lo que el hablante cree acerca de cómo son las cosas y qué significa lo que dice. Así, en la traducción de lenguajes ajenos, podemos obtener su significado si conjeturamos que el hablante cree aproximadamente lo mismo que nosotros, dada la evidencia compartida, 0 , al contrario, dada su conducta verbal, suponiendo un significado común, podemos interpretar sus creencias. Pero siempre, afirma Davidson, debemos conceder al hablante un mundo compartido con nosotros de creencias o de significados ${ }^{8}$. La imposibilidad sistemática de traducción es una lamentable posibilidad, mucho mayor para el historiador, pero lo que el historiador no puede afirmar sin pagar el precio de la incoherencia es que ha comprendido la conducta ajena (es decir, sus creencias acerca del mundo) y que, sin embargo, fracasa sistemáticamente en la traducción de algunos términos.

Un argumento similar al de Davidson lo encontramos en Razón, verdad e historia de Putnam, quien acusa igualmente al relativismo

\footnotetext{
"A esta concesión la denominamos normalmente "Principio de Caridad" y, según Davidson y varios otros autores, es una constricción a priori sobre nuestras capacidades interpretativas o de traducción. Es una forma de conceder al hablante la misma racionalidad que nos concedemos a nosotros mismos. Sobre la mayor o menor fuerza de este principio me he ocupado en Broncano, 1995.
} 
kuhniano o bien de autorrefutarse 9 , o bien de caer en la incoherencia más clara ${ }^{10}$. La posición de Putnam, al igual que la de Davidson, puede ser leída metodológicamente como una constricción a priori para el historiador: a menos que éste asuma una comunidad esencial con otra cultura ajena, sea en términos de significados compartidos, sea en términos de creencias, sea en términos de racionalidad de las decisiones, será incapaz de llevar a cabo su tarea. De manera que habría que concluir que la tarea del historiador, a diferencia de lo que piensa Kuhn, no puede ser completamente autónoma, en el sentido de que las narraciones se autorregulan, por el contrario, siempre dependerá de lo que podemos llamar un compromiso con una cierta descripción cultural o lingüística, la de la "única cultura que tenemos" o del "único lenguaje que hablamos", que no tienen por qué coincidir, claro está, con un lenguaje o cultura en particular, sino con lo que comparten todos los lenguajes o culturas para ser lenguajes $o$ culturas. La historia seguiria siendo, pues, velis nolis esclava de la filosofía.

Otra versión, ligeramente diferente, es la de Kitcher, 1978. Kitcher argumenta que el problema de la inconmensurabilidad o el del relativismo, es decir, el problema de "vivir en mundos diferentes", surge de que al cambiar los significados cambia la referencia de los objetos. Pues bien, afirma Kitcher, lo que podemos hacer es reconstruir el sentido de las teorías o paradigmas pasados, reconstruyéndolas en nuestro lenguaje, y dejar la referencia entre paréntesis. Así, afirma Kitcher, "flogisto es lo que refiere a..." y, seguidamente, relatamos la traducción en nuestro lenguaje de la teoría anterior. La reconstrucción de Kitcher

\footnotetext{
9 "si (...) estuviera en lo cierto -afirma Putnam- sólo podríamos caracterizar a los miembros de otras culturas, incluyendo a los científicos del siglo diecisiete, como animales que producen respuestas a estímulos (incluyendo entre éstos a aguellos ruidos que, curiosamente, se parecen al inglés o al italiano", Putnam, 1981, p.120.

10 "Decir que Galileo poseería nociones que son "inconmensurables" con las nuestras, para seguidamente describirlas con detalle, es algo totalmente incoherente", Putnam, 1981, p.120.
} 
representa en el terreno de la historia lo que en el terreno de la reconstrucción lógica es una oración Ramsey, en la que las aserciones existenciales se separan claramente de la estructura de relaciones y propiedades de los términos de la teoría. De hecho, afirma Kitcher, es lo que en la práctica hace Kuhn cuando actúa como historiador, de manera que se las estaría arreglando muy bien para hacer algo que considera imposible hacer en teoría. La traducción, pues, sería la normal para los casos en los que compartimos la referencia, pero en los casos extraños quedaría en blanco, en un sentido similar a como queda en blanco la referencia de los deícticos cuando no compartimos el contexto del hablante. Por ejemplo cuando alguien dice "ése es el libro que más me ha gustado", pero nosotros no podemos ver hacia donde señala. Para nosotros tal libro sería "el libro que más le gusta a nuestro contertulio". La completa traducibilidad es por consiguiente innecesaria, según Kitcher, para asegurar la comunicabilidad.

Pero es que, además, la completa traducibilidad podría ser insuficiente. Tal es el argumento que desde un punto de vista sociológico, sensiblemente diferente al de Kitcher, ha desarrollado Biagioli, 1990. El argumento de Biagioli es que en algunos casos la traducibilidad completa - que puede estar asegurada incluso por el bilingüismo de alguno de los hablantes - puede no sólo ser insuficiente para la comunicabilidad, sino que, al contrario, podría formar parte de una estrategia abierta de conflicto. Biagioli nos recuerda el caso de Galileo en sus disputas con los aristotélicos acerca de los cuerpos flotantes. Parte de la discusión es lingüística. Galileo acusa a los aristotélicos de que son incapaces de entenderle. Mientras que él es capaz de leer y entender a Aristóteles, se queja Galileo, ellos son incapaces de leer y entender cualquier texto y argumento que esté expresado en lenguaje matemático ${ }^{11}$.

${ }^{11}$ Es curioso que el propio Kuhn emplee este mismo argumento contra los historiadores generales a quienes acusa de ser incapaces de escribir historia de la ciencia porque simplemente son incapaces de leer y entender los textos científicos. Los historiadores, aduce Kuhn con cierta crueldad, son una comunidad que regularmente se nutre de gente que odia las matemáticas. Vide Kuhn, 1971, p.176. 
Biagioli interpreta la actitud galileana como una actitud claramente agresiva destinada a impedir más que a asegurar la comunicación. El contexto es que los aristotélicos le acusaban de emplear el término momento en un sentido incomprensible para ellos y Galileo, quien aduce que él emplea el término en el sentido de la Mecánica, les acusa de ser incapaces de entenderlo. Los aristotélicos, según Biagioli, verian la conducta de Galileo como una conducta claramente invasiva desde otra comunidad diferente. El argumento de Biagioli es que la pertenencia a una comunidad no entraña solamente el compartimiento de elementos cognitivos sino también cierto ethos sin el cual sería imposible una completa comunicabilidad. La traducibilidad estaría siendo empleada desde el punto de vista de Galileo como una estrategia dentro de un conflicto sociológico de disciplinas, aunque ocurra que la disciplina a la que pertenece Galileo sea todavía una disciplina que le tiene a él casi como único miembro.

De ser correcto el argumento de Biagoli, las consideraciones acerca del Principio de Caridad nos llevan aún más lejos, puesto que implican la subordinación del intérprete, sea historiador o participante en una discusión científica, a estrategias de carácter social de un alcance que probablemente no estaria dispuesto a compartir Kuhn, pues nos encontraríamos con que la historia es ahora ancilla sociologiae. Y esto es algo de lo que Kuhn, aún si con menos insistencia que con la Filosofía, quiere evitar.

Tenemos así varias formas de Principio de Caridad, o si queremos expresarlo de otro modo, de constricciones sobre nuestra capacidad hermenéutica, que tienen como consecuencia común convertir a la Historia como práctica de la memoria en un instrumento de alguna otra instancia superior. Para defender la autonomía de la Historia de la Ciencia Kuhn necesita distanciarse de las críticas recibidas al tiempo que tiene que mantener alguna forma de Principio de Caridad si no quiere ser culpable de las acusaciones recibidas. 
Aprender lenguajes y escribir historias

La estrategia kuhniana es alejarse del origen mismo de todas esas críticas, que el sitúa en el prejuicio de que interpretar es lo mismo que traducir. El historiador interpreta, pero no traduce. Es un prejuicio que comparten Davidson, Putnam, y Kitcher que se remonta a Quine ${ }^{12}$. Se trata de una crítica sobre el concepto lingüístico, oracionalista más bien, que tienen del conocimiento que se supone debe reconstruir el historiador. Las diferencias superficiales son claras:

" la traducción es algo efectuado por una persona que sabe dos idiomas [...] el traductor sustituye sistemáticamente palabras o secuencias de palabras en el otro idioma, a fin de producir un texto equivalente." Kuhn, 1983, p. 103.

mientras que la interpretación,

" Es una empresa exigida por la historia y la antropología, entre otras disciplinas. A diferencia de la persona que traduce, puede que la que efectúa la interpretación domine inicialmente sólo una lengua." Kuhn, 1983, p. 104.

La interpretación, ha sido la tesis sostenida por Kuhn con posterioridad a las metáforas gestaltistas, es más bien como aprender una nueva lengua que desconocemos, y

"aprender una nueva lengua no es lo mismo que traducir de ella a la propia. Tener éxito en lo primero no implica que también se vaya a tener éxito en lo segundo [...] Quine está equivocado porque confunde ambos casos." Kuhn, 1983 , p. 105 y 106.

A diferencia de los cambios de Gestalt, la nueva metáfora que comienza a emplear Kuhn a partir de ahora es la del historiador

12 "El argumento (..) depende esencialmente de la ecuación entre interpretación y traducción. Se puede seguir la pista de esta ecuación, como mínimo hasta Palabra y objeto de Quine", Ruhn, 1983, p.102. 
"como intérprete y maestro del lenguaje", y ésta es la habilidad que desarrolla en su trabajo de constructor de narraciones,

¿Cómo puede comunicar sus resultados un historiador que enseña la teoría del flogisto o escribe sobre ella? ¿Qué ocurre cuando presenta a los lectores un grupo de enunciados como aquellos acerca del flogisto que vimos en el epítome anterior? La respuesta a esta pregunta depende del tipo de audiencia, y comenzaré con el que me parece más relevante en este momento: consta de personas que no saben nada de la teoría del flogisto. El historiador les describe el mundo en que creía el químico del siglo XVIII que aceptaba la teoría del flogisto. Simultáneamente les está enseñando el lenguaje que los químicos del siglo XVIII usaban para describir, explicar y explorar ese mundo." Kuhn, 1983, p. 117.

Cuando investiga, pues, aprende un nuevo lenguaje, cuando escribe la narración, lo enseña. Pero lo interesante y lo que debe preocuparnos de este cambio de metáfora es el razonamiento que le lleva a Kuhn a esta separación puesto que lo que no está haciendo es reinventando la hermenéutica ${ }^{13}$. ¿Qué es lo que Kuhn quiere salvar al diferenciar la operación de interpretar de la de traducir? La respuesta está en la diferencia entre el conocimiento que redescubre el historiador y el conocimiento que usa el traductor. Las constricciones son diferentes, el traductor establece una proyección desde términos u oraciones actuales de un texto o acto de habla desde un lenguaje a otros. El núcleo esencial es aquí la "actualidad", es decir, en el hecho de que el contexto está cerrado por el texto o el contexto conversacional presente ante el narrador o el lector. Para cumplir esta tarea quizás emplee alguna dosis de interpretación, reconoce Kuhn, pero el intérprete hace algo más, reconstruye o reconstituye un conjunto de potencialidades que no están presentes en el registro histórico y que tal vez ni siquiera se hayan actualizado históricamente. La interpretación descubre o debe descubrir el uso de los términos y el uso o

13 "Yo no soy, como algunos lectores de La estructura de las revoluciones cietíficas han pensado, un mistico o un hombre que descubre que nociones como intuición, Verstehen o empatía son útiles a la filosofía de la ciencia en su forma actual", Kuhn, 1974, "Respuesta al Prof. Sylvain" p. 562. 
conocimiento práctico no se agota en su uso efectuado hasta el momento. Si fuese así la tarea del historiador sería la de un documentalista sin ninguna función cognitiva de descubrimiento. El intérprete o historiador debe redescubrir algo que ya está esencialmente oculto, que son las expectativas de uso.

Tanta importancia tiene esta cuestión metodológica que desde 1983, cuando escribió "Conmensurabilidad, comparabilidad y comunicabilidad", la preocupación de Kuhn por los temas de lenguaje, tal como se muestra al menos en el espacio dedicado a ellos en sus escritos, se ha hecho obsesiva. En lo que desgraciadamente han sido hasta ahora sus "Afterwords", en Kuhn, 1993, deja ya de preocuparle incluso el aspecto metafórico ligado al aprendizaje de una nueva lengua para dejar claro que su preocupación es por el mecanismo subyacente:

"Aunque ya no hablaré más de algo tan vago como "cambio de lenguaje" sí hablaré de cambios de conceptos y de sus nombre, de vocabulario conceptual y de lexicón conceptual estructurado que contiene tanto conceptos de clase como sus nombres." Kuhn, 1993 p. 316.

La importancia de los términos de clase es crucial en el conocimiento, son los que establecen la ontología básica y los que expresan las diferencias que el científico puede establecer en el mundo. Las características que nos interesan son

(1) "Los términos de clase se aprenden en el uso: alguien ya adepto a su uso proporciona al aprendiz ejemplos de su aplicación apropiada." Kuhn, 1993, p. 316.

(2) "Esa característica presenta una segunda propiedad de los términos de clase: son proyectables: conocer cualquier término de clase es conocer algunas generalizaciones satisfechas por sus referentes y estar equipado para buscar otras." Kuhn, 1993, p. 312.

(3) "las expectativas adquiridas al aprender un término, aunque pueden diferir de individuo a individuo, proporcionan al individuo que las ha adquirido el significado del término. Cambios en las expectativas sobre los referentes de un término de clase son por consiguiente cambios en su significado." Kuhn, 1993, p. 317. 
El aprendizaje como aprendizaje de uso, la proyectabilidad basada en el dominio de las generalizaciones y las expectativas son las tres características que determinan que el vocabulario específico de una narración histórica sea la reconstrucción de un conocimiento que, en primer lugar, es potencial y no se reduce a ninguna operación de recuperación del registro histórico y, en segundo lugar, relacionado con lo anterior, se trata de un conocimiento perdido. Es por ello por lo que, respondiendo a la propuesta de Kitcher, de usar una oración Ramsey para dejar entre paréntesis la referencia de términos de nuestro pasado científico, afirma Kuhn,

"dar con el referente de un término definido mediante el enunciado Ramsey no sirve de ninguna ayuda para determinar el refenente de la siguiente ocurrencia de ese término." Kuhn, 1983, p. 120.

De manera que la interpretación, como operación diferente de la traducción y fundamento de la reconstrucción de la memoria, lo es en la medida en que transciende al lenguaje, por cuanto el narrador debe recuperar algo que transciende al lenguaje, las formas como otra gente del pasado relacionaba el lenguaje con el mundo. De ahí que la narración sea una operación de recuperación de "mundos perdidos", que lo son en el doble sentido de no haber permanecido a lo largo de la historia y en el de estar ocultos tras las prácticas abiertas de los científicos del pasado y pertenecer a su historia cognitiva, a los modelos mentales que les capacitaban para emplear con éxito el lenguaje como instrumento de investigación, pero sobre todo de cohesión en su comunidad particular.

Queda la duda razonable de si esta estrategia kuhniana evita verdaderamente el alcance de las críticas desde el Principio de Caridad. Al separar Kuhn la interpretación de la traducción lo hace no tanto sobre una base metodológica de principio, como la que encontramos habitualmente entre los filósofos que se alinean en la corriente hermenéutica, sino, desde mi punto de vista, desde una teoría del contenido alternativa a la puramente lingüística de Davidson. De acuerdo a esta concepción, muy esquemáticamente, la línea del contenido cognitivo está trazada por la barrera del 
lenguaje, de manera que si no podemos traducir es que no podemos entender. Kuhn habría extendido la noción habitual de contenido mediante su teoría de los paquetes de términos que se aprenden en la práctica. A Kuhn le preocupan las operaciones cognitivas que conectan el lenguaje con el mundo, y no solamente los aspectos puramente intralingüisticos; y desde esta perspectiva habría un cierto conocimiento práctico que no queda reflejado en la formulación lingüistica. Esta idea, como es sabido, se remonta a $\mathrm{M}$. Polanyi, en parte a Wittgenstein, y aparece recogida de manera parcial y confundente en la ecuación de "significado como uso", ecuación que nos remitiría de nuevo al lenguaje, puesto que el uso se entiende básicamente como uso intralingüístico. Pero Kuhn piensa que el lenguaje tiene sentido en un mundo de prácticas cognitivas de clasificación y categorización que tienen una estructura parcialmente independiente del lenguaje $y$, por consiguiente, quedan reflejadas solamente de manera parcial en la traducción. Una reconstrucción completa implicaria, por el contrario, recobrar al menos el dominio medio del saber práctico implicado en el dominio léxico. Si convivimos con el otro grupo, cual es el caso de un traductor radical, podemos lograr esta recuperación mediante la observación directa de los hablantes en su relación con el mundo. En el caso del historiador que ya no tiene acceso a la práctica de los agentes, se pone en marcha la operación que Kuhn ha denominado "interpretación", que exige recorrer hacia atrás ese camino cognitivo hasta recuperar parcialmente las prácticas perdidas.

No obstante las diferencias con Davidson y Putnam, Kuhn se ve obligado a sostener una cierta forma de Principio de Caridad que se expresaría como una constricción de coherencia sobre las narraciones. Tal constricción sólo es observada por el historiador profesional, en el mejor sentido de experto en narraciones. Tan sólo su conocimiento práctico $\mathrm{y}$, no alguna supuesta regla formal de método, le permite juzgar con criterios internos la validez de una narración. Por ejemplo, cuando se enfrenta a los éxitos y fracasos de los científicos del pasado, 
"La habilidad para explicar estos éxitos y fracasos es básica para la interpretación de los textos que efectúa el historiador de la ciencia. (Si una interpretación atribuye al autor de un texto aserciones repetidas que observaciones fácilmente obtenibles hubieran debilitado, entonces es casi seguro que la interpretación es errónea, y el historiador debe comenzar su trabajo de nuevo)." Kuhn, 1983 p. 109-10, nota 8.

De manera que el historiador se mueve en un espacio de posibilidades internas con las que construye su narración. Podríamos aducir que estas posibilidades son las que están potencialmente en la semántica de las teorías, tal como expresaría la imagen tradicional de la tarea del historiador, y como ejemplo recordemos la perspectiva lakatosiana de recuperar estas posibilidades como posibilidades lógicas que, en notas al margen, se corrigen como actualidades psicológicas ${ }^{14}$ pero, este es mi argumento, nos alejaríamos mucho de lo que constituye a mi modo de ver la novedad de Kuhn, la de fundar el trabajo del historiador sobre un objetivo de descubrimiento, no de mera reconstrucción, un descubrimiento que es dificil en la medida en que se realiza entre tensiones insoslayables. Veamos cuáles son, y marginalmente tendrá también respuesta la observación de Biagioli.

\section{Las tensiones esenciales del historiador}

Una de las consecuencias de nuestra reconstrucción de Kuhn es la imposibilidad de leer sus tesis en clave trivialmente sociologista, bien sea porque se suponga que los intereses no epistémicos, sociales, externos determinan el contenido de "otros mundos" que recuperamos en la historia, bien sea en el sentido más interesante de que el nivel de las relaciones y estructura social determina el contenido del nivel cognitivo del aprendizaje $e^{15}$. La autonomía de

14 Lakatos, 1971. Son varios los autores que han señalado que Kuhn está menos alejado de lo que parece de las tesis de la Filosofía de la Ciencia, incluso de su versión paradigmática representada por Carnap.

${ }^{15}$ La fuerza de la tendencia que nos lleva a leer a Kuhn en clave sociológica es 
las narraciones históricas se sostiene sobre un difícil equilibrio que el saber práctico del historiador tiene que reconstruir entre tres tensiones esenciales

*Tensión primera: innovación y continuidad

*Tensión segunda: individuo y comunidad

*Tensión tercera: contenido interno y externo

La primera tensión da título al segundo libro teórico de Kuhn. Todas se reducen a ella. ¿Cómo es posible la creatividad? ¿Cómo es posible reconstruir algo intrínsecamente histórico y contingente, cual es un descubrimiento? Si la continuidad es la regla, como cabría esperar de una lectura superficial del Kuhn más popular, la novedad sería un milagro dada la presión del paradigma, la ciencia normal, el reconocimiento de la comunidad. Pero la novedad es posible: las revoluciones son una consecuencia de la novedad, no sólo su causa. En momentos de baja productividad de un paradigma, un suceso contingente como la creación individual modifica la historia. Pues bien, solamente podemos situar esta creatividad en un territorio que se sitúa, si no equidistante, sí al menos constituido por la tensión de los otros dos polos: por un lado el individuo, por otro la comunidad que le reconoce como

muy grande. Es cierto que en Kuhn 1970a se afirma "ya debe estar claro que, en última instancia, la explicación deberá ser psicológica o sociológica. Es decir, deberá ser la descripción de un sistema de valores, una ideología, junto con un análisis de las instituciones mediante las cuales se transmite e impone este sistema", p. 314. Una página más adelante reconoce coincidir con Popper sobre la irrelevancia del individuo, aunque señala la existencia de "un largo paso del rechazo de la idiosincrasia psicológica del individuo al rechazo de los elementos comunes inducidos por la educación y el adiestramiento dentro de la conformación psicológica del miembro titulado del grupo científico" p. 315. En el prólogo a 1977, matiza, sin embargo, la lectura: "mi trabajo tiene profundas raices sociológicas, pero no de una manera que permita separar el tema de la epistemología", Kuhn, 1977 pg. 21, y más adelante, "Cuando hablo aquí de historia de la ciencia me refiero a esa parte primordial del campo que se refiere a la evolución de las ideas científicas, sus métodos y técnicas, no a esa parte cada vez más importante que hace hincapié en el status social de la ciencia, en especial los cambiantes patrones de educación científica, la institucionalización y el apoyo, tanto moral como económico". Kuhn, 1976, p. 36. 
experto; por un lado sus modelos mentales individuales e idiosincrásicos, por otra las prácticas abiertas que tienen que alcanzar un éxito reconocible, predictivo, técnico y, sobre todo, comunicativo.

En esta confrontación ocurren elementos superficiales, como son los que postula Biagioli, estrategias más o menos exitosas de comunicación o simplemente de poder, es cierto, pero las tensiones esenciales son mucho mas profundas e interesantes. El Kuhn más cercano a sí mismo como historiador, el que nos desvela el nudo de tensiones lo encontramos en dos lugares. En la teoría deja entrever estas tensiones en una conferencia un tanto coloquial que lee en 1968 ( Kuhn , 1977, págs. 27-45), "Las relaciones entre la Historia y la Filosofía de la Ciencia". Allí describe en un tono inusualmente intimista los duros problemas que tiene que resolver el historiador una vez que ya tiene toda la información encima de la mesa, y los compara con el trabajo del filósofo, para quien la investigación previa sólo es un pretexto, a veces innecesario, para aclarar sus ideas. Pero es en lo que me parece su obra magna, La teoría del cuerpo negro y la discontinuidad cuántica, 1894-1912 donde Kuhn, al reconstruir el descubrimiento de Plank, nos muestra mejor en la práctica que en cualquier otro texto teórico la constelación de tensiones que debe resolver un autor para dar lugar a una transformación tan radical como el pensamiento cuántico. Plank tenía que resolver un conflicto interno, casi insoportable, entre modelos clásicos mecánicos, que estaban aún presentes en el tratamiento de los procesos estadísticos debido a Maxwell, y lo que comenzaba a a puntar Boltzmann de una manera muy embrionaria, que medidas y términos esencialmente estadísticos podían tener referentes físicos. Es aquí, en la búsqueda de referentes para términos que social (disciplina riamente) no podían referir, más que a entidades puramente matemáticas, donde la tensión de la narración de Kuhn refleja, esa es mi creencia, una correspondiente, paralela y refleja tensión en el proceso de descubrimiento de Plank, una tensión que hace de la narración un objeto singular que recobra algo esencialmente perdido, el hecho del descubrimiento 
como algo singular, contingente en sí mismo, pero necesario para nuestra propia identidad.

Recordamos porque lo que queremos recuperar no está presente y porque su recuperación es importante para nosotros. Recordamos porque hemos perdido parte de nuestra identidad, en este caso, de nuestra identidad cognitiva, y recordamos porque esa identidad solamente existe en tanto que proceso histórico, contingente, del que somos producto y otros fueron productores. Hemos comenzado el trabajo con una cita bien conocida de Borges. Escrita en los años cuarenta es una mera reivindicación del lector en la construcción del contenido de la narración, leída en los años noventa, después de la post-modernidad filosófica y literaria, significa el sorprendente, insólito descubrimiento de la insoportable tensión del historiador entre la identidad y la memoria. 


\section{BIBLIOGRAFIA*}

BIAGIOLI, M. (1990), "The Antropology of Inconmensurability". Studies in History and Philosophy of Science, 21: 183-209.

BronCano, F. (1995), "La fuerza del PC (Principio de Caridad). Circunstancia, habilidad y contenido en la explicación de la acción". Anélisis, $n^{2}$ monográfico sobre Filosofía de la Mente. (Próxima publicación.)

DAVIDSON, D. (1974), "On the Very Idea of a Conceptual Scheme". Proceedings and Addresses of the American Philosophical Association, 47, 5-20; v. esp. 'Sobre la idea misma de un esquema conceptual", en De la verdad y la interpretación, Barcelona: GEDISA, 1994.

KITCHER, P. (1978), "Theories, Theorists and Theoretical Change". The Philosophical Review, 87: 519-47.

KUHN, T. S. (1968), "La Historia de la Ciencia" en Kuhn, 1977. (1970)a, "Lógica del descubrimiento o psicología de la investigación", en I. Lakatos y A. Musgrave (eds.) Crítica y conocimiento, Barcelona, Crítica, 1973.

(1970)b, La estructura de las reooluciones científicas, $2^{\mathrm{a}}$ ed., México: Fondo de Cultura Económica, 1971.

(1971), "Las relaciones entre la Historia y la Historia de la Ciencia", en Kuhn, 1977.

(1974), "Segundos pensamientos sobre los paradigmas" y "discusión", en

F. Suppe, (ed.), La estructura de las teorías cientificas, Madrid: Editora Nacional.

(1976), "La Historia y la Filosofía de la Ciencia", en Kuhn, 1977.

(1977), La tensión esencial. Estudios selectos sobre la tradición y el cambio científico, México: Fondo de Cultura Económica, 1982.

(1978), La teoría del cuerpo negro y la discontinuidad cuántica, 1894-1912, Madrid, Alianza Editorial, 1980.

(1983), "Conmensurabilidad, comparabilidad y comunicabilidad", en ¿Qué son las reooluciones cientificas y otros ensayos, Bancelona: Paidós, 1989.

(1993), "Afterwords", en P. Horwich (ed.), World Changes, Cambridge, Mass.: MIT Press.

LAKATOS, I. (1971), "La historia de la ciencia y sus reconstrucciones racionales", en G. Currie ed. La metodología de los programas de investigación, Madrid: Alianza Editorial. 1983.

PUTNAM, H. (1981), Razón, verdad e historia, Madrid, Tecnos, 1988.

* Habida cuenta de que las traducciones de Kuhn al español son de uso común, se cita por dichas versiones; sin embargo la fecha de referencia es la de la publicación en el original inglés, salvo en La estructura de las reooluciones científicas, que es la de la segunda edición inglesa sobre la que se realizó la versión española. 